\title{
The Daidzein Metabolite, 6,7,4'-Trihydroxyisoflavone, Is a Novel Inhibitor of PKCa in Suppressing Solar UV-Induced Matrix Metalloproteinase 1
}

Tae-Gyu Lim ${ }^{1,2,3, \dagger}$, Jong-Eun Kim ${ }^{1,2,3, \dagger}$, Sung-Young Lee ${ }^{2}$, Jun Seong Park ${ }^{4}$, Myung Hun Yeom ${ }^{4}$, Hanyong Chen ${ }^{2}$, Ann M. Bode ${ }^{2}$, Zigang Dong ${ }^{2, *}$ and Ki Won Lee 1,3,5,*

1 World Class University Biomodulation Major, Department of Agricultural Biotechnology and Center for Food and Bioconvergence, Seoul National University, Seoul 151-742, Korea; E-Mails: tglim83@gmail.com (T.-G.L.); idonlike@gmail.com (J.-E.K.)

2 The Hormel Institute, University of Minnesota, Austin, MN 55912, USA; E-Mails: slee@hi.umn.edu (S.-Y.L.); hchen@hi.umn.edu (H.C.); bodex008@umn.edu (A.M.B.)

3 Advanced Institutes of Convergence Technology, Seoul National University, Suwon 443-270, Korea

4 Skin Research Institute, Amorepacific Corporation R\&D Center, Yongin 446-829, Korea; E-Mails: superbody@amorepacific.com (J.S.P.); mhyeom@amorepacific.com (M.H.Y.)

5 Research Institute of Bio Food Industry, Institute of Green Bio Science and Technology, Seoul National University, Pyeongchang 232-916, Korea

$\dagger$ These authors contributed equally to this work.

* Authors to whom correspondence should be addressed;

E-Mails: zgdong@hi.umn.edu (Z.D.); kiwon@snu.ac.kr (K.W.L.);

Tel.: +1-507-437-9600 (Z.D.); +82-2-880-4661 (K.W.L.);

Fax: +1-507-437-9606 (Z.D.); +82-2-878-6178 (K.W.L.).

External Editor: Terrence Piva

Received: 10 September 2014; in revised form: 31 October 2014 / Accepted: 4 Novermber 2014 / Published: 19 November 2014

\footnotetext{
Abstract: Soy isoflavone is an attractive source of functional cosmetic materials with anti-wrinkle, whitening and skin hydration effects. After consumption, the majority of soy isoflavones are converted to their metabolites in the human gastrointestinal tract. To understand the physiological impact of soy isoflavone on the human body, it is necessary to evaluate and address the biological function of its metabolites. In this study, we investigated the effect of 6,7,4'-trihydroxyisoflavone (6,7,4'-THIF), a major metabolite of
} 
daidzein, against solar UV (sUV)-induced matrix metalloproteinases (MMPs) in normal human dermal fibroblasts. MMPs play a critical role in the degradation of collagen in skin, thereby accelerating the aging process of skin. The mitogen-activated protein/extracellular signal-regulated kinase (MEK)/extracellular signal-regulated kinase (ERK), mitogen-activated protein kinase (MKK)3/6/p38 and MKK4/c-Jun $N$-terminal kinases (JNK) signaling pathways are known to modulate MMP-1 function, and their activation by sUV was significantly reduced by $6,7,4^{\prime}$-THIF pretreatment. Our results also indicated that the enzyme activity of protein kinase $\mathrm{C}(\mathrm{PKC}) \alpha$, an upstream regulator of MKKs signaling, is suppressed by $6,7,4^{\prime}$-THIF using the in vitro kinase assay. Furthermore, the direct interaction between $6,7,4^{\prime}-$ THIF and endogenous PKC $\alpha$ was confirmed using the pull-down assay. Not only sUV-induced MMP-1 expression, but also sUV-induced signaling pathway activation were decreased in PKC $\alpha$ knockdown cells. Overall, we elucidated the inhibitory effect of 6,7,4'-THIF on sUV-induced MMPs and suggest PKC $\alpha$ as its direct molecular target.

Keywords: matrix metalloproteinase 1; protein kinase $\mathrm{C}(\mathrm{PKC}) \alpha$; photoaging; 6,7,4'-trihydroxyisoflavone

\section{Introduction}

With the continued increase of the elderly population in modern society, the demand for ways to look younger is also rising. The prevention of skin aging is vital for maintaining a younger-looking appearance [1]. Accordingly, preventing or reversing skin aging has become a central subject of research in the dermatological and cosmeceutical fields [2]. Skin aging is a naturally occurring process of senescence and is accompanied by clinical signs, such as sagging, fine wrinkles and paleness $[3,4]$. Genetic and environmental factors contribute to the aging of skin. In particular, exposure to sunlight is a key factor contributing to the acceleration of the skin aging process [5]. Skin that is excessively exposed to sunlight shows a decline of skin functions and features deep wrinkles, dryness and uneven pigmentation [4,6,7], which is a process termed photoaging [1,8,9]. Ultraviolet (UV) light is mainly responsible for the skin damaging effect of sunlight. Recent research is focusing on developing strategies to prevent UV-induced biochemical changes in the skin, thereby alleviating skin photoaging.

Human skin tissue is composed of various extracellular matrix (ECM) components, such as collagen, keratin and elastin. Skin wrinkles are primarily formed as a result of collagen breakdown. While various enzymes participate in the degradation of ECM, matrix metalloproteinases (MMPs) are the key enzymes for degrading ECM with substrate specificity [10-12]. Among them, MMP-1 is the primary enzyme that degrades collagen in human skin $[13,14]$. Although various environmental factors can activate MMP-1 expression, solar UV (sUV) is a major inducer of MMP-1 expression in skin [12]. Thus, suppression of sUV-induced MMP-1 expression is regarded as a powerful strategy for development of anti-photoaging agents.

Multiple lines of evidence have reported the health benefits of soy isoflavones [15-18]. In particular, daidzein has been shown to have a beneficial effect on skin health [19] and has a high amount of daily intake [20]. 6,7,4'-trihydroxyisoflavone (6,7,4'-THIF, Figure 1A) is one of the major derivatives converted from daidzein [21]. We previously reported the pharmacological activities of 
daidzein derivatives $[15,16,18]$, including the anti-cancer effects of 7,3',4'-THIF and 6,7,4'-THIF in skin and colon. The anti-atopic dermatitis effect of 7,3',4'-THIF was reported in the NC/Nga Mice model [22]. However, the effect of 6,7,4'-THIF and its molecular target in skin aging have not been elucidated yet.

In the present study, we aimed to investigate the anti-skin photoaging effect of 6,7,4'-THIF. We showed that 6,7,4'-THIF attenuated sUV-induced MMP-1 expression more effectively than its precursor, daidzein, by directly suppressing protein kinase $\mathrm{C}(\mathrm{PKC}) \alpha$ kinase activity, suggesting its potential protective effect against skin photoaging.

Figure 1. Molecular structure of 6,7,4'-trihydroxyisoflavone $\left(6,7,4^{\prime}\right.$-THIF) (A) and daidzein (B).

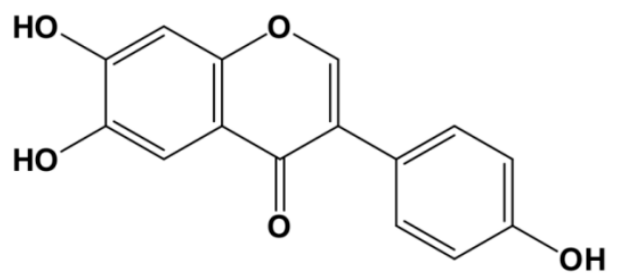

6,7,4'-Trihydroxyflavone (THIF)

(A)

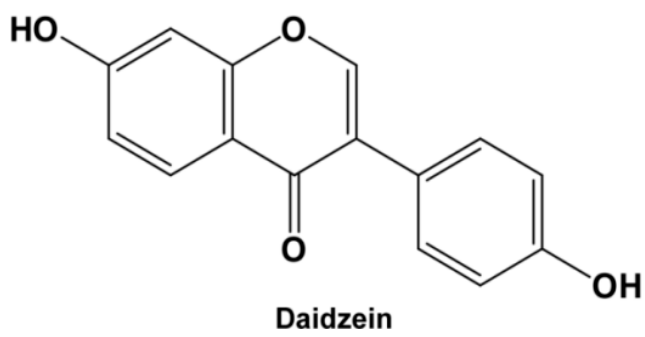

(B)

\section{Results}

\subsection{6,7,4'-Trihydroxyisoflavone (6,7,4'-THIF) Reduces Solar UV (sUV)-Induced Matrix} Metalloproteinase (MMP)-1 Expression in Normal Human Dermal Fibroblasts (NHDF) Cells

Accumulative data have shown that UV radiation induces MMP-1 expression, which is a key responsible mechanism in UV-induced skin wrinkle formation [12,13,23,24]. Hence, the inhibition of MMP-1 expression serves as an excellent strategy in developing anti-wrinkle agents. We compared the inhibitory effect of daidzein and its metabolite, 6,7,4'-THIF, on sUV-induced MMP-1 expression in normal human dermal fibroblasts (NHDF) cells. MMP-1 expression was markedly elevated by sUV irradiation, and 6,7,4'-THIF suppressed sUV-induced MMP-1 expression in a dose-dependent manner (Figure 2A). On the other hand, daidzein did not lead to a significant reduction of sUV-induced MMP-1 expression at the concentration used in this study (10 or $20 \mu \mathrm{M})$ in NHDF cells. Daidzein and $6,7,4$ '-THIF did not exhibit cytotoxicity at the concentration we used in this study (Figure 2B). Taken together, 6,7,4'-THIF shows a better inhibitory effect on sUV-induced MMP-1 expression than its precursor, daidzein.

\subsection{6,7,4'-THIF Inhibits sUV-Induced Mitogen-Activated Protein Kinase(MAPK) Signaling Pathways} in NHDF Cells

MMP-1 is regulated by various inflammatory signaling pathways, including the MAPK pathways [25,26]. To determine if MAPKs are involved in the inhibitory function of 6,7,4'-THIF on sUV-induced MMP-1 expression, we evaluated the effect of 6,7,4'-THIF on sUV-induced MAPK phosphorylation in NHDF cells. Similar to previous studies, phosphorylation of MAPKKs and MAPKs was induced by sUV exposure, and 6,7,4'-THIF reduced sUV-induced upregulation of the MEK-ERK, 
MKK4-JNK and MKK3/6-p38 signaling pathways (Figure 3). As all of the MAPKK phosphorylation was decreased by 6,7,4'-THIF in NHDF cells, we hypothesized that 6,7,4'-THIF may regulate an upstream modulator of MAPKKs.

Figure 2. 6,7,4'-THIF suppresses solar UV (sUV)-induced MMP-1 expression in normal human dermal fibroblasts (NHDF) cells with a non-cytotoxic concentration range. (A) To determine the non-cytotoxic concentration range of the compounds, [3-(4,5-dimethylthiazol-2-yi)-5-(3carboxymethoxyphenyl)-2-(4-sulfophenyl)-2H-tetrazolium, inner salt] (MTS) analysis was performed. The cells were cultured to confluence in 96-well plates, and then, the cells were treated for $24 \mathrm{~h}$ with daidzein and 6,7,4'-THIF $(1,10,20,40,80$ and $100 \mu \mathrm{M})$. The cell viability was determined using MTS analysis, as described in the Materials and Methods; and (B) 6,7,4'-THIF shows a better inhibitory effect than its precursor, daidzein, on sUV-induced MMP-1 expression. After $1 \mathrm{~h}$ of treatment with each compound, the cells were exposed to sUV. The secreted MMP-1 was measured using western blot analysis, as described in the Materials and Methods. Data are representative of three independent experiments, which gave similar results. Means with different letters $(\mathrm{a}-\mathrm{c})$ within a graph are significantly different from each other at $p<0.05$.

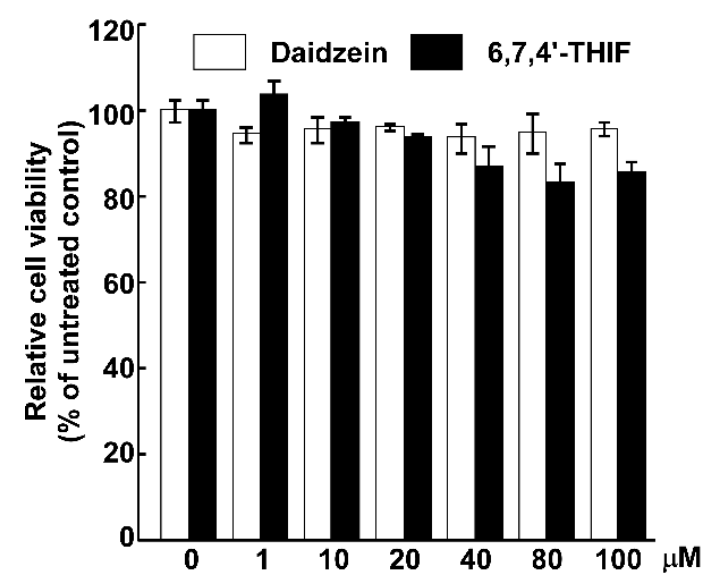

(A)

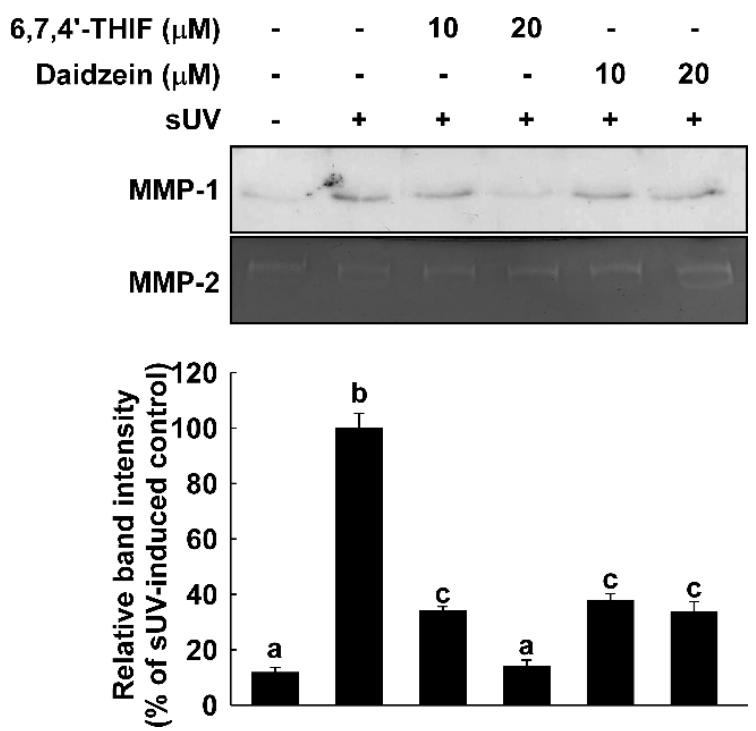

(B)

\subsection{6,7,4'-THIF Suppresses Protein Kinase C (PKC) $\alpha$ Kinase Activity with Direct Binding}

PKC is a well-known upstream regulator of MAPKKs. Recently, several papers have indicated that PKC $\alpha$ mediates MMP-1 expression by activating various signaling pathways [23,27]. Additionally, PKC kinase activity and the protein level of $\mathrm{PKC} \alpha$ in human skin fibroblasts from older donors is increased compared to that of younger donors, suggesting its potential association with the skin aging process [27]. Thus, we hypothesized that PKC $\alpha$ is a potential target of $6,7,4^{\prime}$-THIF in the regulation of MMP-1 expression. In Figure 4A, although sUV exposure enhanced phosphorylation of PKC $\alpha, 6,7,4^{\prime}$-THIF did not affect sUV-induced PKC $\alpha$ phosphorylation. Next, we evaluated the effect of 6,7,4'-THIF on PKC $\alpha$ kinase activity. Treatment with 6,7,4'-THIF suppressed the kinase activity of PKC $\alpha$ in a dose-dependent manner (Figure 4B), whereas PKC $\delta$ kinase activity was not significantly suppressed by 6,7,4'-THIF. To 
verify if 6,7,4'-THIF directly interacts with $\mathrm{PKC} \alpha$, an ex vivo pull-down assay was conducted using 6,7,4'-THIF conjugated with Sepharose 4B in NHDFs cell lysate. Our result showed that 6,7,4'-THIF directly binds with PKC $\alpha$ in cell lysate, and this interaction was not diminished by ATP (Figure 4D). Overall, we demonstrated that 6,7,4'-THIF inhibits sUV-induced MMP-1 expression by directly suppressing PKC $\alpha$ kinase activity.

Figure 3. 6,7,4'-THIF down-regulates sUV-induced MAPKK and MAPK activation. To determine the effect 6,7,4'-THIF on the sUV-induced MAPKK (A) and MAPK (B) signaling pathway, the phosphorylation level of the signaling proteins was analyzed by western blot, as described in the Materials and Methods. After pretreatment with 6,7,4'-THIF (10 and $20 \mu \mathrm{M})$ for $1 \mathrm{~h}$, the cells were exposed to sUV. The cell lysates were collected after an additional 15 min (p-MEK, p-MKK3/6 and p-MKK4) and 30 min (p-ERK, p-p38 and p-JNK). Data are representative of three independent experiments, which gave similar results. Means with different letters $(\mathrm{a}-\mathrm{c})$ within a graph are significantly different from each other at $p<0.05$.

A
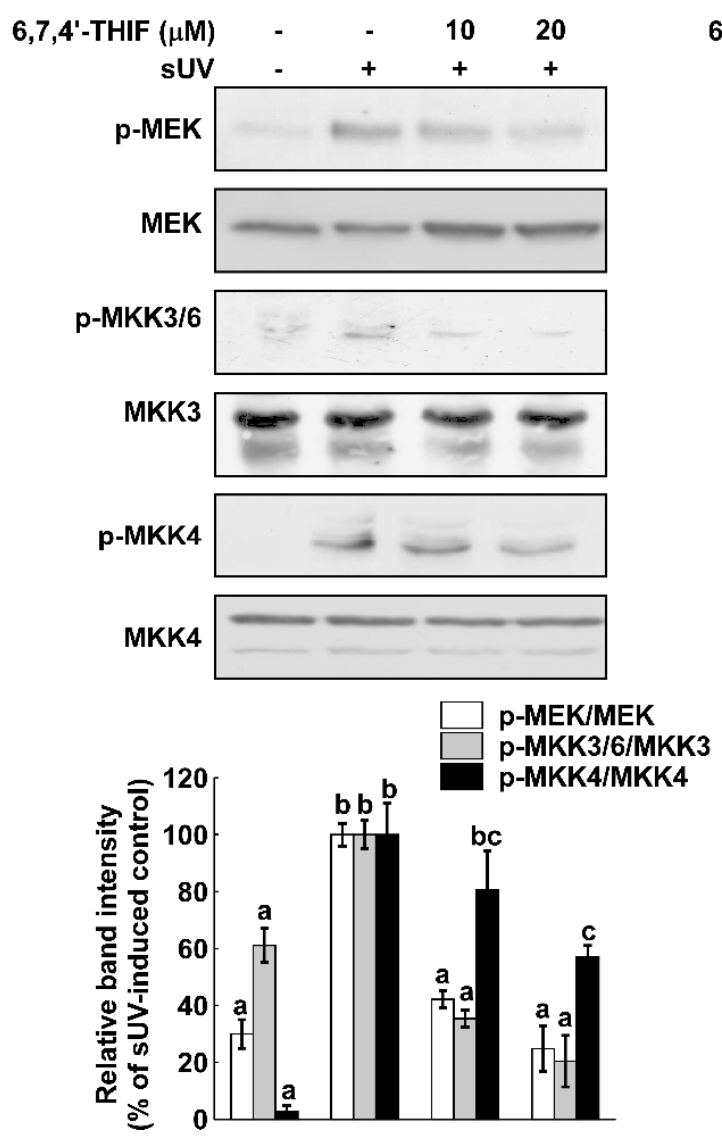

B

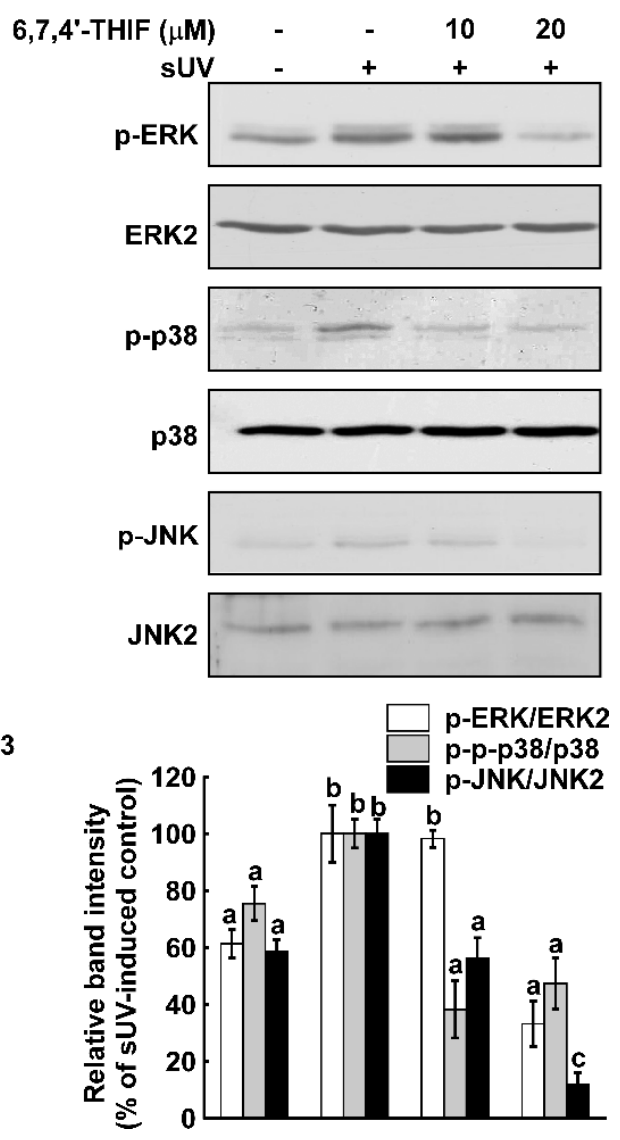

\subsection{PKCa Plays a Crucial Role in sUV-Induced MMP-1 Expression in NHDFs}

Among PKC isoforms, the increased protein level of $\mathrm{PKC} \alpha$ was observed in older donor's skin fibroblasts [27]. Hence, we determined whether PKC $\alpha$ is closely related in sUV-induced MMP-1 expression in the current model using shPKC $\alpha$-NHDFs. While the MMP-1 expression level was increased by sUV irradiation in shMock, the exposure of sUV did not induce MMP-1 expression in shPKC $\alpha$-NHDFs (Figure 5A). To determine whether PKC $\alpha$ regulates the sUV-induced MAPK signaling 
pathway, we evaluated the phosphorylation levels of MAPKs in shPKC $\alpha$-NHDFs. Contrary to the results in shMock-NHDFs, the MEK-ERK, MKK4-JNK and MKK3/6-p38 signaling pathways were not activated by sUV irradiation in shPKC $\alpha$-NHDFs (Figure 5B-D). Taken together, PKC $\alpha$ plays a critical role in sUV-induced MMP-1 expression as an upstream regulator of the MEK-ERK, MKK4-JNK and MKK3/6-p38 signaling pathways in NHDFs.

Figure 4. PKC $\alpha$ is a direct target of 6,7,4'-THIF. (A) The cells were pretreated with 6,7,4'-THIF for $1 \mathrm{~h}$ and irradiated with sUV. After $5 \mathrm{~min}$, the cells were lysed, and the phosphorylated PKC $\alpha$ level was evaluated; (B) Each active $\mathrm{PKC} \alpha$ and $\mathrm{PKC} \delta$ was co-incubated with $6,7,4^{\prime}-\mathrm{THIF}$ at the indicated concentrations for $30 \mathrm{~min}$ at $30^{\circ} \mathrm{C}$, then MBP, a substrate of PKC $\alpha$ and PKC $\delta$, and $\left[\gamma_{-}{ }^{32} \mathrm{P}\right]$-ATP were added to the mixture and additionally incubated for $10 \mathrm{~min}$. The incorporated radiolabeled phosphate was measured using a scintillation counter. Data are represented as the means $\pm \mathrm{SD}$, as determined from three independent experiments. Means with different letters $(\mathrm{a}-\mathrm{c})$ within a graph were significantly different from each other at $p<0.05$; (C) 6,7,4'-THIF directly binds to endogenous PKC $\alpha$. The binding of 6,7,4'-THIF with PKC $\alpha$ was visualized using immunoblotting with a specific PKC $\alpha$ antibody: Lane 1 (input control), whole-cell lysates from NHDF; Lane 2 (control), lysates from NHDF; and Lane 3, whole-cell lysates from NHDF cells precipitated with 6,7,4'-THIF-Sepharose 4B beads; and (D) 6,7,4'-THIF does not compete with ATP for binding with PKC $\alpha$. Active PKC $\alpha$ was incubated with ATP at different concentrations $(10,100$ and $1000 \mu \mathrm{M})$ with $100 \mu \mathrm{L}$ of 6,7,4'-THIF-Sepharose 4B beads or $100 \mu \mathrm{L}$ of Sepharose 4B (as a negative control) overnight. After washing, the binding was confirmed by western blot.

A

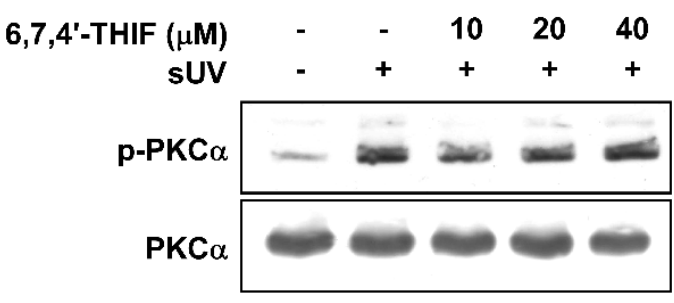

C

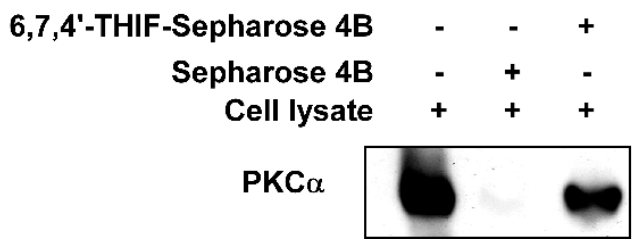

B

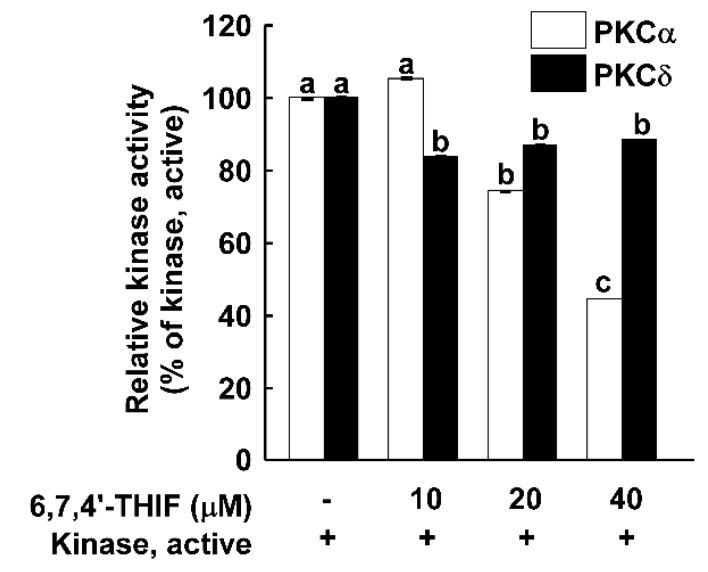

D

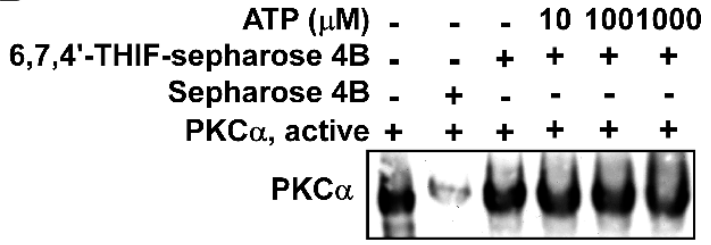


Figure 5. $\mathrm{PKC} \alpha$ modulates $\mathrm{sUV}$-induced $\mathrm{MMP}-1$ expression by activation of the MAPK signaling pathway. (A) sUV could not induce MMP-1 expression in shPKC $\alpha$-NHDF cells compared with shMock-NHDF cells. After $48 \mathrm{~h}$ of sUV irradiation, the media were collected, and the MMP-1 expression was analyzed using western blot analysis, as described in the Materials and Methods; PKC $\alpha$ regulates the sUV-induced MEK/ERK (B), MKK4/JNK (C) and MKK3/6/p38 (D) signaling pathways. After starvation with serum-free DMEM for $24 \mathrm{~h}$, shMock and shPKC $\alpha$-NHDF cells were exposed to sUV or not. Then, the protein was harvested after 15 min (p-MEK, p-MKK4 and p-MKK3/6) or $30 \mathrm{~min}$ (p-ERK, p-JNK and p-p38). The level of protein was visualized using specific antibodies.

A
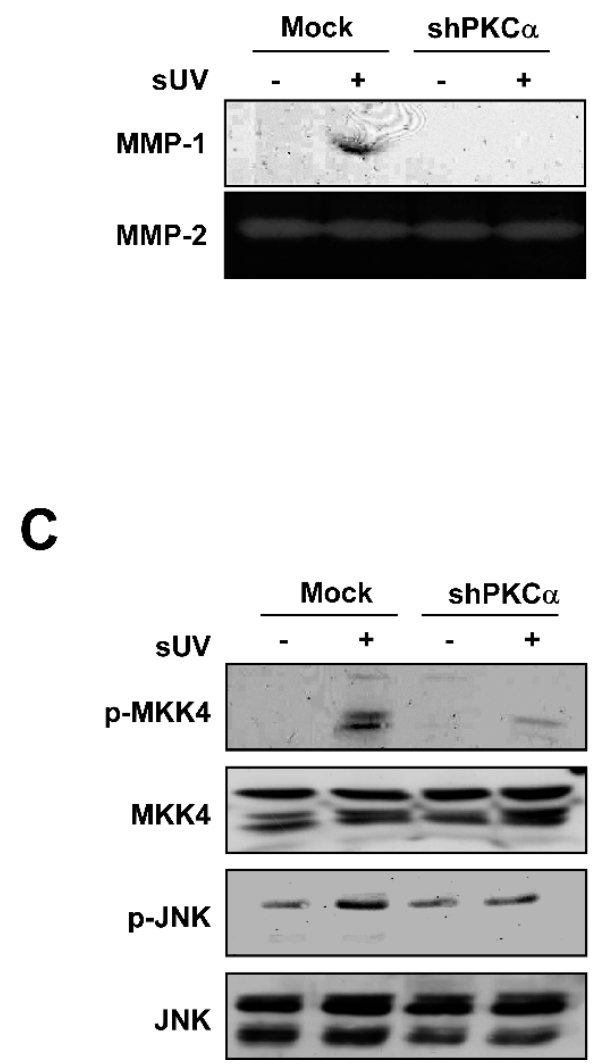

B

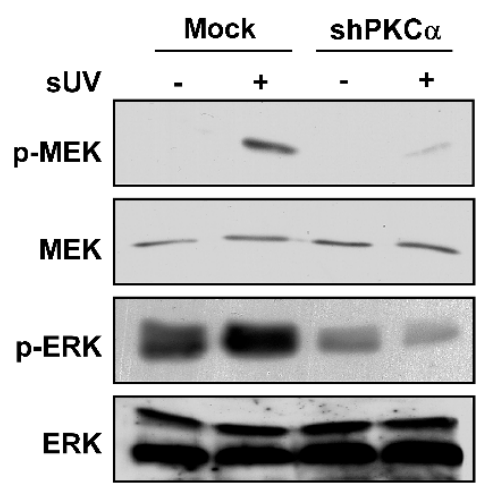

D

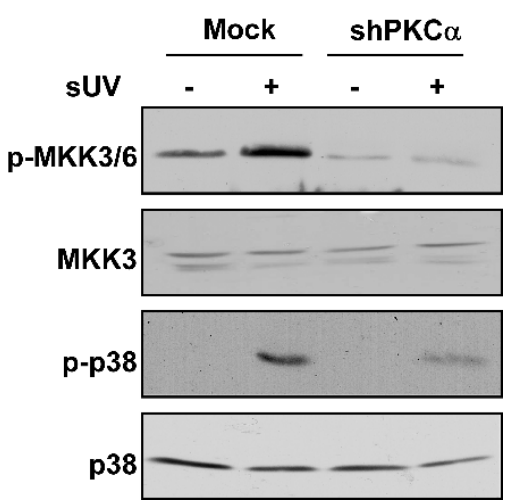

\subsection{Discussion}

Soybeans are an attractive source for cosmetics due to their health benefits and safety [28]. Various cosmetic products have been developed incorporating soybean ingredients, such as soybean fiber, peptide and isoflavone [29]. As a differentiation strategy in the market, companies use fermentation and bioconversion technology to enhance the effect and value of soybean materials [21]. Since many types of fermented soybean foods have been used for a long time and various methods of fermentation and bioconversion have already been developed, the fermentation and bioconversion of soybeans is relatively easier than other sources. During the fermentation and bioconversion of soybeans, isoflavone, which is a major active component in soybeans, is converted to a variety of derivatives [30]. 6,7,4'-THIF is one of the derivatives converted from daidzein, which is a well-known soy isoflavonoid [21]. Oxidation of daidzein at the six carbon of the A ring generates 6,7,4'-THIF, which is medicated by the 3 cytochrome P450 enzyme, including CYP1A2, CYP1A1 and CYP1B1 [31]. 6,7,4'-THIF could be produced industrially by 
bioconversion using these enzymes from daidzein. If the anti-aging effects of 6,7,4'-THIF are verified, $6,7,4$ '-THIF will be a valuable cosmetic material.

A previous study reported daidzein's potential as an anti-skin aging agent [19]. Although daidzein did not show an inhibitory effect on the sUV-induced MMP-1 expression level in our results (Figure 2A), its bioconversion product, 6,7,4'-THIF, significantly suppressed sUV-induced MMP-1 expression. This result is along the same lines with previous literature showing the improved bioactive function of bioconverted soybean products and compounds. Lee et al., have shown that fermented soybean has a stronger inhibitory effect on MAPKs than raw soybean [32]. Additionally, 6,7,4'-THIF revealed a better inhibitory effect on MMP-1 expression than retinoic acid, an industrial anti-wrinkle ingredient (Figure 6). This notion indicated that 6,7,4'-THIF can be a new anti-skin aging compound. We previously showed that another daidzein metabolite, 7,3',4'-THIF, is more effective at inhibiting Ultravioloet B (UVB)-induced cyclooxygenase-2 (COX-2) expression than daidzein [16].

We suggested that PKC $\alpha$ is a direct molecular target of 6,7,4'-THIF on sUV-induced MMP-1 expression. Direct inhibition of PKC $\alpha$ by 6,7,4'-THIF reduced sUV-induced MAPK signaling and MMP-1 expression. PKC $\alpha$, a member of the serine/threonine kinase PKC family, has been implicated in various biological activities, such as cell survival and proliferation [33-40]. Interestingly, PKC activity is increased in fibroblasts with age. Total PKC activity was shown to be four-times higher in human skin fibroblasts of 61-year-old donors than those of one-week-old donors [27]. This study also showed that only the $\mathrm{PKC} \alpha$ protein level is increased with age using specific antibodies against $\mathrm{PKC}$ isoforms $(\alpha, \delta, \varepsilon$ and $\zeta)$. In accordance with these results, the protein level of $\mathrm{PKC} \alpha$ is up-regulated in aged in vitro cell.

$\mathrm{PKC} \alpha$ is expressed in human keratinocytes, and the inhibition of PKC using pharmacological inhibitors suppressed heat-induced MMP-1 expression [23]. The decreased MMP-1 expression was shown in siPKC $\alpha$-HaCaT, but not in siPKC $\beta$ - or siPKC $\delta$-HaCaT cells. The parallel expression level of MMP-1 with the PKC $\alpha$ protein level was also reported. Indeed, our data showed that sUV could not induce MMP-1 expression in shPKC $\alpha$-NHDF. In addition, the MEK/ERK, MKK4/JNK and MKK3/6/p38 signaling pathways were not activated by UV in shPKC $\alpha$-NHDF cells. Overall, we confirmed that PKC $\alpha$ plays a critical role in MMP-1 expression by sUV irradiation by activating the MEK/ERK, MKK4/JNK and MKK3/6/p38 signaling pathways. Furthermore, we revealed that $\mathrm{PKC} \alpha$ is the direct target of $6,7,4$ '-THIF by demonstrating the inhibition of PKC $\alpha$ kinase activity by $6,7,4$ '-THIF and the direct interaction of 6,7,4'-THIF with PKC $\alpha$ in cell lysate. We also elucidated that 6,7,4'-THIF binds to the non-ATP binding site of PKC $\alpha$. The result of ATP-independent binding of 6,7,4'-THIF to PKC $\alpha$ indicates that 6,7,4'-THIF can inhibit PKC $\alpha$ kinase activity in an allosteric manner. However, to further verify how 6,7,4'-THIF interacts with $\mathrm{PKC} \alpha$, a structural study is required utilizing X-ray crystallography and computer modeling. Additionally, although our data support the biological activity and mechanisms of $6,7,4^{\prime}$-THIF in vitro, in vivo and clinical studies are needed to further validate its anti-skin aging effect.

Overall, we demonstrated that 6,7,4'-THIF may exert an anti-photoaging effect by suppressing MMP-1, which plays a key role in wrinkle formation. In addition, we revealed that the inhibitory effect of 6,7,4'-THIF on MMP-1 results from its direct interaction with and suppression of PKC $\alpha$. 
Figure 6. 6,7,4'-THIF shows a better inhibitory effect than retinoic acid on sUV-induced MMP-1 expression. The cells were pretreated with 6,7,4'-THIF for $1 \mathrm{~h}$ and irradiated with sUV. The media was collected after $48 \mathrm{~h}$ of sUV exposure. The MMP-1 expression level was measured by western blot analysis as described in the Materials and Methods. Means with different letters $(\mathrm{a}-\mathrm{c})$ within a graph were significantly different from each other at $p<0.05$.

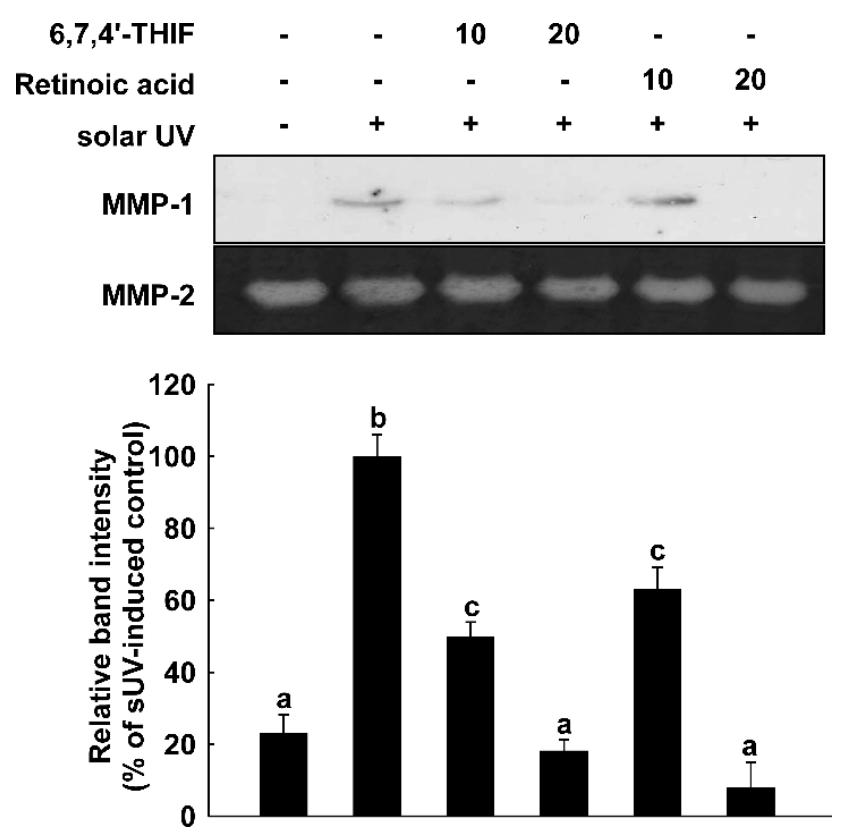

\section{Materials and Methods}

\subsection{Materials}

6,7,4'-THIF was obtained from Chromadex ${ }^{\mathrm{TM}}$ (Irvine, CA, USA), and Dulbecco's modified eagle medium (DMEM) and MMP-1 antibody were purchased from Thermo Fisher Scientific (San José, CA, USA). Medium 199 (M199) and daidzein were purchased from Sigma-Aldrich (St. Louis, MO, USA), and fetal bovine serum (FBS) was purchased from Gemini Bio-Products (Calabasas, CA, USA). CNBr-Sepharose 4B, [ $\gamma-32 \mathrm{P}]$-ATP and the chemiluminescence detection kit were obtained from Amersham Pharmacia Biotech (Piscataway, NJ, USA). The protein assay kit was purchased from Bio-Rad Laboratories (Hercules, CA, USA). MTS solution was from Promega (Madison, WI, USA). Penicillin/streptomycin was purchased from Invitrogen (Grand Island, NY, USA). Primary antibodies recognizing phosphorylated MEK ( $\operatorname{Ser}^{217 / 221}$ ), total MEK, phosphorylated SEK1/MKK4 (MKK4, $\left.\operatorname{Ser}^{257} / \mathrm{Thr}^{261}\right)$, phosphorylated MKK3 $\left(\operatorname{Ser}^{189}\right) / 6\left(\operatorname{Ser}^{207}\right)$, total MKK3, phosphorylated p38 (Tyr $\left.{ }^{180 / 182}\right)$, total p38 and PKC $\alpha$ were purchased from Cell Signaling Technology (Danvers, MA). Antibodies against phosphorylated-ERKs $\left(\mathrm{Tyr}^{204}\right.$ ), total ERKs, ERK2, total MKK4, phosphorylated JNK ( $\mathrm{Thr}^{183} / \mathrm{Tyr}^{185}$ ), total JNK, JNK2 and phosphorylated PKC $\alpha\left(\mathrm{Ser}^{657}\right)$ were obtained from Santa Cruz Biotechnology (Santa Cruz, CA, USA). Active PKC $\alpha$ and PKC $\delta$ proteins were purchased from Millipore (Bedford, MA, USA). 


\subsection{Cell Culture}

Normal human dermal fibroblasts (NHDF) cells were obtained from the American Type Culture Collection (Manassas, VA, USA). The cells were cultured in DMEM/M199 (4:1) supplemented with $10 \%(v / v)$ FBS under $37^{\circ} \mathrm{C}, 5 \% \mathrm{CO}_{2}$ conditions.

\subsection{Cell Cytotoxicity}

To examine the cytotoxicity of daidzein and 6,7,4-THIF on NHDF cells, the cells were seeded in 96-well plates. Upon reaching confluency, the cells were starved with serum-free DMEM for $24 \mathrm{~h}$. After incubation with the various doses of daidzein or 6,7,4-THIF for $24 \mathrm{~h}$, the cytotoxicity of the compounds was analyzed with Cell Titer 96 Aqueous One Solution (Promega). In brief, the cells were incubated with $20 \mathrm{~mL}$ of MTS solution for $1 \mathrm{~h}$ at $37{ }^{\circ} \mathrm{C}$ in a $5 \% \mathrm{CO}_{2}$ incubator. The absorbance was evaluated at $492 \mathrm{~nm}$.

\subsection{Solar UV Irradiation}

The solar UV resources are UVA-340 lamps (Q-Lab Corporation; Cleveland, OH, USA). The region of the wavelength is from 295 to $365 \mathrm{~nm}$ with the major peak emission of $340 \mathrm{~nm}$. The percentage of UVA and UVB of the sUV source was measured using a UV meter as $94.5 \%$ and $5.5 \%$, respectively. The dose of sUV used in the current study was $60 \mathrm{~kJ} / \mathrm{m}^{2}$. For sUV irradiation, the media were changed with serum-starved DMEM/M199 (4:1) before sUV exposure. Then, the plate cover was taken off for direct sUV irradiation of the cells.

\subsection{Western Blot Analysis}

NHDF cells were cultured to confluence and starved with serum-free DMEM for $24 \mathrm{~h}$. Additionally, the $6,7,4^{\prime}-$ THIF was added to NHDF at various doses $(10$ and $20 \mu \mathrm{M})$ for $1 \mathrm{~h}$. The cells were exposed to sUV, subsequently. Equal amounts of proteins were separated on 10\% SDS-polyacrylamide gels. Additionally, the proteins were transferred to Immobilon P membranes (Millipore). The membranes were blocked with $5 \%$ fat-free milk for $1 \mathrm{~h}$ and incubated with specific primary antibodies at $4{ }^{\circ} \mathrm{C}$ overnight. Next, the proteins were hybridized with HRP-conjugated secondary antibody, and the light emission was detected using a chemiluminescence detection kit (GE Healthcare, Pittsburgh, PA, USA).

\subsection{Lentiviral Infection}

To generate the knocked down PKC $\alpha$ of NHDF, a shRNA system was used. The lentiviral expression vectors, including Gipz-shPKC $\alpha$ (RNAi core, University of Minnesota, Minneapolis, MN, USA), and packaging vectors, including pMD2.0G and psPAX, were purchased from Addgene Inc. (Cambridge, MA, USA). To prepare shPKC $\alpha$ viral particles, each viral vector and the packaging vectors (pMD2.0G and psPAX) were transfected into HEK293T cells using jetPEI following the manufacturer's instructions. The transfection medium was changed at $4 \mathrm{~h}$ after transfection, and the cells were then cultured for $36 \mathrm{~h}$. The viral particles were harvested by filtration using a $0.45-\mathrm{mm}$ syringe filter, then combined with $8 \mu \mathrm{g} / \mathrm{mL}$ polybrene (EMD Millipore) and infected into 60\% confluent NHDF cells overnight. The cell culture medium was replaced with fresh complete growth medium for $24 \mathrm{~h}$ before 
the cells were selected for using puromycin (Sigma, St Louis, MO, USA, $2 \mu \mathrm{g} / \mathrm{mL}$ ) over $36 \mathrm{~h}$. The selected cells were then used for further experiments.

\subsection{Zymography}

The activity of MMP-2 was evaluated using zymography. Zymography was performed using 10\% polyacrylamide gels in the presence of gelatin $(0.5 \mathrm{mg} / \mathrm{mL})$ as a substrate for MMP-2. The samples were suspended in loading buffer (10\% SDS, 25\% glycerol, $0.25 \mathrm{M}$ Tris (pH 6.8) and $0.1 \%$ bromophenol blue) and loaded onto 10\% SDS-PAGE gels without denaturation. After electrophoresis, the gels were incubated in renaturing buffer (Invitrogen) at room temperature for $30 \mathrm{~min}$ and then incubated for $24 \mathrm{~h}$ at $37^{\circ} \mathrm{C}$ in developing buffer (Invitrogen). The gels were then stained with $0.5 \%$ Coomassie Brilliant Blue.

\subsection{In Vitro Kinase Assay}

PKC $\alpha$ and $\delta$ kinase assays were performed using active recombinant $\mathrm{PKC} \alpha$ and $\delta$ enzymes following the manufacturer's instructions. Briefly, active PKC $\alpha$ or $\delta$ and $6,7,4^{\prime}-\mathrm{THIF}$ were incubated at $30{ }^{\circ} \mathrm{C}$ for $15 \mathrm{~min}$ in assay buffer (20 mM MOPS (pH 7.2), $25 \mathrm{mM} \beta$-glycerol phosphate, $5 \mathrm{mM}$ EGTA, $1 \mathrm{mM}$ sodium orthovanadate and $1 \mathrm{mM}$ DTT). Two millimeters of myelin basic protein (MBP) substrate were added to each mixture, then incubated at $30{ }^{\circ} \mathrm{C}$ for $15 \mathrm{~min}$ with $[\gamma-32 \mathrm{P}]$-ATP solution in a magnesium acetate-ATP cocktail buffer (Upstate Biotechnology Inc., Lake Placid, NY, USA). Next, the reaction mixture was transferred onto p81 paper. Using $0.75 \%$ phosphoric acid, the p81 paper was washed three times for $5 \mathrm{~min}$. The radio-labeled phosphate was detected using a scintillation counter.

\subsection{6,7,4'-THIF Pull-Down Assay Using Sepharose 4B}

6,7,4'-THIF-Sepharose 4B complex was generated by following the procedure described in the previous literature [41]. The NHDF cell lysate was incubated with this 6,7,4'-THIF-Sepharose 4B complex overnight at $4{ }^{\circ} \mathrm{C}$. The binding of the protein to $6,7,4^{\prime}-\mathrm{THIF}-\mathrm{Sepharose} 4 \mathrm{~B}$ was examined by western blot analysis.

\subsection{ATP and PKCa Competition Assay}

Recombinant PKCa (200 ng) was incubated with ATP (10, 100 and $1000 \mu \mathrm{M})$ at $4{ }^{\circ} \mathrm{C}$ for $1 \mathrm{~h}$. Sepharose 4B (negative control) or 6,7,4'-THIF-Sepharose 4B was added to the mixture and incubated $4{ }^{\circ} \mathrm{C}$ overnight. Extra proteins were washed with wash buffer, and $\mathrm{PKC} \alpha$ was detected by western blot analysis.

\subsection{Statistical Analysis}

Data were expressed as the means \pm standard deviation (SD). One-way analysis of variance (ANOVA) with Tukey's HSD test was used to evaluate mean differences of group and statistical significance. Differences were considered significant at $p<0.05$. 


\section{Acknowledgments}

This work was supported by the National Leap Research Program (2010-0029233) of the National Research Foundation funded by the Ministry of Science, ICT and Future Planning, the High Value-added Food Technology Development Program (311035-3) funded by the Ministry of Agriculture, Food and Rural Affairs, and the R\&D program (N0000697) of MOTIE/KIAT (Establishment of Infra Structure for Anti-aging Industry Support) of Republic of Korea.

\section{Author Contributions}

Jun Seong Park, Myung Hun Yeom, Ann M. Bode, Zigang Dong and Ki Won Lee conceived of and designed the experiments; Tae-Gyu Lim, Sung-Young Lee and Hanyong Chen performed the experiments; and Jong-Eun Kim analyzed the data and wrote the paper.

\section{Conflicts of Interest}

The authors declare no conflict of interest.

\section{References}

1. Sjerobabski-Masnec, I.; Situm, M. Skin aging. Acta Clin. Croat. 2010, 49, 515-518.

2. Gunn, D.A.; Rexbye, H.; Griffiths, C.E.; Murray, P.G.; Fereday, A.; Catt, S.D.; Tomlin, C.C.; Strongitharm, B.H.; Perrett, D.I.; Catt, M.; et al. Why some women look young for their age. PLoS One 2009, 4, e8021.

3. Yaar, M.; Gilchrest, B.A. Photoageing: Mechanism, prevention and therapy. Br. J. Dermatol. 2007, $157,874-887$.

4. Jenkins, G. Molecular mechanisms of skin ageing. Mech. Ageing Dev. 2002, 123, 801-810.

5. Uitto, J. Understanding premature skin aging. N. Engl. J. Med. 1997, 337.

6. Yaar, M. Clinical and Histological Features of Intrinsic Versus Extrinsic Skin Aging; Springer: Berlin, Germany, 2006.

7. Chung, J.H. Photoaging in asians. Photodermatol. Photoimmunol. Photomed. 2003, 19, 109-121.

8. Hwang, K.A.; Yi, B.R.; Choi, K.C. Molecular mechanisms and in vivo mouse models of skin aging associated with dermal matrix alterations. Lab. Anim. Res. 2011, 27, 1-8.

9. Oh, J.H.; Kim, Y.K.; Jung, J.Y.; Shin, J.E.; Kim, K.H.; Cho, K.H.; Eun, H.C.; Chung, J.H. Intrinsic aging- and photoaging-dependent level changes of glycosaminoglycans and their correlation with water content in human skin. J. Dermatol. Sci. 2011, 62, 192-201.

10. Visse, R.; Nagase, H. Matrix metalloproteinases and tissue inhibitors of metalloproteinases: Structure, function, and biochemistry. Circ. Res. 2003, 92, 827-839.

11. Lauer-Fields, J.L.; Juska, D.; Fields, G.B. Matrix metalloproteinases and collagen catabolism. Biopolymers 2002, 66, 19-32.

12. Brennan, M.; Bhatti, H.; Nerusu, K.C.; Bhagavathula, N.; Kang, S.; Fisher, G.J.; Varani, J.; Voorhees, J.J. Matrix metalloproteinase-1 is the major collagenolytic enzyme responsible for collagen damage in UV-irradiated human skin. Photochem. Photobiol. 2003, 78, 43-48. 
13. Kim, M.K.; Lee, D.H.; Lee, S.; Kim, E.J.; Chung, J.H. Uv-induced DNA damage and histone modification may involve $M M P-1$ gene transcription in human skin in vivo. J. Dermatol. Sci. 2014, 73, 169-171.

14. Ho, J.N.; Lee, Y.H.; Park, J.S.; Jun, W.J.; Kim, H.K.; Hong, B.S.; Shin, D.H.; Cho, H.Y. Protective effects of aucubin isolated from eucommia ulmoides against UVB-induced oxidative stress in human skin fibroblasts. Biol. Pharm. Bull. 2005, 28, 1244-1248.

15. Lee, D.E.; Lee, K.W.; Jung, S.K.; Lee, E.J.; Hwang, J.A.; Lim, T.G.; Kim, B.Y.; Bode, A.M.; Lee, H.J.; Dong, Z. 6,7,4'-Trihydroxyisoflavone inhibits hct-116 human colon cancer cell proliferation by targeting CDK1 and CDK2. Carcinogenesis 2011, 32, 629-635.

16. Lee, D.E.; Lee, K.W.; Byun, S.; Jung, S.K.; Song, N.; Lim, S.H.; Heo, Y.S.; Kim, J.E.; Kang, N.J.; Kim, B.Y.; et al. 7,3',4'-Trihydroxyisoflavone, a metabolite of the soy isoflavone daidzein, suppresses ultraviolet b-induced skin cancer by targeting cot and MKK4. J. Biol. Chem. 2011, 286, $14246-14256$.

17. Liu, S.; Hsieh, D.; Yang, Y.L.; Xu, Z.; Peto, C.; Jablons, D.M.; You, L. Coumestrol from the national cancer institute's natural product library is a novel inhibitor of protein kinase CK2. BMC Pharmacol. Toxicol. 2013, 14, 36.

18. Seo, S.G.; Yang, H.; Shin, S.H.; Min, S.; Kim, Y.A.; Yu, J.G.; Lee, D.E.; Chung, M.Y.; Heo, Y.S.; Kwon, J.Y.; et al. A metabolite of daidzein, 6,7,4'-trihydroxyisoflavone, suppresses adipogenesis in 3T3-L1 preadipocytes via ATP-competitive inhibition of PI3K. Mol. Nutr. Food Res. 2013, 57, $1446-1455$.

19. Zhao, D.; Shi, Y.; Dang, Y.; Zhai, Y.; Ye, X. Daidzein stimulates collagen synthesis by activating the TGF- $\beta$ /smad signal pathway. Australas. J. Dermatol. 2014, doi:10.1111/ajd.12126.

20. Nakamura, Y.; Tsuji, S.; Tonogai, Y. Determination of the levels of isoflavonoids in soybeans and soy-derived foods and estimation of isoflavonoids in the japanese daily intake. J. AOAC Int. 2000, 83, 635-650.

21. Goh, M.J.; Park, J.S.; Bae, J.H.; Kim, D.H.; Kim, H.K.; Na, Y.J. Effects of ortho-dihydroxyisoflavone derivatives from korean fermented soybean paste on melanogenesis in B16 melanoma cells and human skin equivalents. Phytother. Res. 2012, 26, 1107-1112.

22. Kim, B.B.; Kim, J.R.; Kim, J.H.; Kim, Y.A.; Park, J.S.; Yeom, M.H.; Joo Lee, H.; Lee, K.W.; Kang, N.J. 7,3',4'-Trihydroxyisoflavone ameliorates the development of dermatophagoides farinae-induced atopic dermatitis in NC/Nga mice. Evid.-Based Complement. Altern. Med. 2013, 2013, 636597.

23. Lee, Y.M.; Li, W.H.; Kim, Y.K.; Kim, K.H.; Chung, J.H. Heat-induced MMP-1 expression is mediated by TRPV1 through PKC $\alpha$ signaling in HaCaT cells. Exp. Dermatol. 2008, 17, 864-870.

24. Lim, T.G.; Jung, S.K.; Kim, J.E.; Kim, Y.; Lee, H.J.; Jang, T.S.; Lee, K.W. NADPH oxidase is a novel target of delphinidin for the inhibition of UVB-induced MMP-1 expression in human dermal fibroblasts. Exp. Dermatol. 2013, 22, 428-430.

25. Ma, Y.S.; Hsiao, Y.P.; Lin, J.H.; Hsu, S.C.; Chueh, F.S.; Weng, S.W.; Lai, K.C.; Lin, J.G.; Chung, J.G. Crude extract of Rheum palmatum L. inhibits migration and invasion of LS1034 human colon cancer cells acts through the inhibition of matrix metalloproteinase-2/-9 by MAPK signaling. Environ. Toxicol. 2014, doi:10.1002/tox.21962. 
26. Wang, Y.; Chen, H.; Wang, W.; Wang, R.; Liu, Z.L.; Zhu, W.; Lian, S. N-Terminal 5-mer peptide analog P165 of amyloid precursor protein inhibits UVA-induced MMP-1 expression by suppressing the MAPK pathway in human dermal fibroblasts. Eur. J. Pharmacol. 2014, 734C, 1-8.

27. Ricciarelli, R.; Maroni, P.; Ozer, N.; Zingg, J.M.; Azzi, A. Age-dependent increase of collagenase expression can be reduced by $\alpha$-tocopherol via protein kinase $\mathrm{c}$ inhibition. Free Radic. Biol. Med. 1999, 27, 729-737.

28. Ravindranath, M.H.; Muthugounder, S.; Presser, N.; Viswanathan, S. Anticancer therapeutic potential of soy isoflavone, genistein. Adv. Exp. Med. Biol. 2004, 546, 121-165.

29. Wilson, R. Soybean: Market driven research needs. In Genetics and Genomics of Soybean; Stacey, G., Ed.; Springer: New York, NY, USA, 2008; Volume 2, pp 3-15.

30. Chen, K.I.; Erh, M.H.; Su, N.W.; Liu, W.H.; Chou, C.C.; Cheng, K.C. Soyfoods and soybean products: From traditional use to modern applications. Appl. Microbiol. Biotechnol. 2012, 96, 9-22.

31. Atherton, K.M.; Mutch, E.; Ford, D. Metabolism of the soyabean isoflavone daidzein by CYP1A2 and the extra-hepatic CYPs 1A1 and 1B1 affects biological activity. Biochem. Pharmacol. 2006, 72, 624-631.

32. Lee, T.H.; Do, M.H.; Oh, Y.L.; Cho, D.W.; Kim, S.H.; Kim, S.Y. Dietary fermented soybean suppresses UVB-induced skin inflammation in hairless mice via regulation of the MAPK signaling pathway. J. Agric. Food Chem. 2014, 62, 8962-8972.

33. Sanchez-Bautista, S.; Nicolas, F.E. Recent patents concerning modulators of protein kinase C. Recent Pat. DNA Gene Seq. 2013, 7, 74-81.

34. Jain, K.; Basu, A. Protein kinase C-E promotes emt in breast cancer. Breast Cancer 2014, 8, 61-67.

35. Chen, Z.; Forman, L.W.; Williams, R.M.; Faller, D.V. Protein kinase C- $\delta$ inactivation inhibits the proliferation and survival of cancer stem cells in culture and in vivo. BMC Cancer 2014, 14, 90.

36. Mochly-Rosen, D.; Das, K.; Grimes, K.V. Protein kinase C, an elusive therapeutic target? Nat. Rev. Drug Dis. 2012, 11, 937-957.

37. Tsao, H.K.; Chiu, P.H.; Sun, S.H. PKC-dependent ERK phosphorylation is essential for P2X 7 receptor-mediated neuronal differentiation of neural progenitor cells. Cell Death Dis. 2013, 4, e751.

38. Do Carmo, A.; Balca-Silva, J.; Matias, D.; Lopes, M.C. PKC signaling in glioblastoma. Cancer Biol. Ther. 2013, 14, 287-294.

39. Hu, C.T.; Cheng, C.C.; Pan, S.M.; Wu, J.R.; Wu, W.S. PKC mediates fluctuant ERK-paxillin signaling for hepatocyte growth factor-induced migration of hepatoma cell HepG2. Cell Signal. 2013, 25, 1457-1467.

40. Cavaliere, F.; Benito-Munoz, M.; Panicker, M.; Matute, C. NMDA modulates oligodendrocyte differentiation of subventricular zone cells through PKC activation. Front. Cell. Neurosci. 2013, 7, 261.

41. Lim, T.G.; Kwon, J.Y.; Kim, J.; Song, N.R.; Lee, K.M.; Heo, Y.S.; Lee, H.J.; Lee, K.W. Cyanidin-3-glucoside suppresses B[a]PDE-induced cyclooxygenase-2 expression by directly inhibiting Fyn kinase activity. Biochem. Pharmacol. 2011, 82, 167-174.

(C) 2014 by the authors; licensee MDPI, Basel, Switzerland. This article is an open access article distributed under the terms and conditions of the Creative Commons Attribution license (http://creativecommons.org/licenses/by/4.0/). 